\title{
Effect of maternal weight, adipokines, glucose intolerance and lipids on infant birth weight among women without gestational diabetes mellitus
}

\author{
Ravi Retnakaran MD, Chang Ye MSc, Anthony J.G. Hanley PhD, Philip W. Connelly PhD, Mathew Sermer MD, \\ Bernard Zinman MD, Jill K. Hamilton MD
}

See related commentary by Ryan on page 1341 and at www.cmaj.ca/lookup/doi/10.1503/cmaj.120682

\begin{abstract}
Background: The delivery of excess maternal nutrients to the fetus is known to increase the risk of macrosomia, even among infants of women without gestational diabetes mellitus. With the current obesity epidemic, maternal adiposity and its associated effects on circulating adipokines and inflammatory proteins may now have a greater impact on fetal growth. We sought to evaluate the independent effects of maternal glycemia, lipids, obesity, adipokines and inflammation on infant birth weight.
\end{abstract}

Methods: We included 472 women who underwent an oral glucose tolerance test in late pregnancy and were found not to have gestational diabetes; 104 (22.0\%) had gestational impaired glucose tolerance. We also measured fasting levels of insulin, low- and high-density lipoprotein cholesterol, triglycerides, leptin, adiponectin and C-reactive protein. Obstetric outcomes were assessed at delivery.

Results: The mean birth weight was $3481 \mathrm{~g}$ (standard deviation $493 \mathrm{~g}$ ); 68 of the infants were large for gestational age. On multiple linear regression analysis, positive determinants of birth weight were length of gestation, male infant, weight gain during pregnancy up to the time of the oral glucose tolerance test, body mass index (BMI) before pregnancy and impaired glucose tolerance in pregnancy. Leptin, adiponectin and C-reactive protein levels were each negatively associated with birth weight. On logistic regression analysis, the significant metabolic predictors of having a large-for-gestational-age infant were BMI before pregnancy (odds ratio [OR] 1.16, $95 \%$ confidence interval [CI] 1.05-1.27, per $1 \mathrm{~kg} / \mathrm{m}^{2}$ increase), weight gain during pregnancy up to the time of the oral glucose tolerance test (OR 1.12, 95\% Cl 1.05-1.19, per $1 \mathrm{~kg}$ increase) and leptin level (OR $0.50,95 \% \mathrm{Cl}$ $0.30-0.82$, per 1 standard deviation change).

Interpretation: Among women without gestational diabetes, maternal adiposity and leptin levels were the strongest metabolic determinants of having a large-for-gestational-age infant rather than glucose intolerance and lipid levels.
I n 1952, Jørgen Pedersen proposed that delivery of excess maternal glucose to the fetus may be responsible for the increased risk of macrosomia among infants of women with diabetes during pregnancy. ${ }^{1} \mathrm{He}$ postulated that maternal hyperglycemia leads to fetal hyperglycemia, which in turn stimulates insulin secretion in the fetus, the anabolic effects of which result in excessive fetal growth. Since its introduction, the Pedersen hypothesis has been further extended by other investigators and accepted as the pathophysiologic basis for increased risk of macrosomia among infants of women with diabetes during pregnancy., ${ }^{2,3}$ Accordingly, for pregnant women with either pre-existing dia- betes or gestational diabetes, modern clinical practice focuses on normalizing blood glucose levels to reduce the risk of fetal hyperglycemia and hence the risk of fetal macrosomia and its associated adverse clinical outcomes (e.g., shoulder dystocia, birth injury, need for cesarean delivery).

It is now recognized that the association between maternal nutrients and fetal growth is not restricted solely to women with diabetes. Several studies have shown associations linking maternal blood glucose and triglyceride levels with infant birth weight among women without gestational diabetes. $^{4-7}$ This awareness has led to recent recommendations to lower the diagnostic thresholds
Competing interests: None declared.

This article has been peer reviewed.

Correspondence to: Jill K. Hamilton, jill.hamilton@sickkids.ca

CMAJ 2012. DOI:10.1503 /cmaj.111154 
for gestational diabetes on glucose tolerance testing in pregnancy, to optimize the detection of women who may be at risk of having a large-forgestational-age infant. ${ }^{8}$

Another important factor relevant to the risk of macrosomia is maternal adiposity. ${ }^{9}$ Indeed, the past decade has seen a marked increase in the prevalence of pre-existing obesity among pregnant women. ${ }^{10}$ In the context of the current obesity epidemic, we hypothesized that, in women without gestational diabetes, maternal adiposity and its associated effects on circulating levels of adipokines (e.g., adiponectin and leptin) and inflammatory proteins (C-reactive protein) may now have a greater impact than glucose and lipid levels on fetal growth. We conducted this study to evaluate the independent effects of maternal glycemia, lipid levels, obesity, adipokine levels and inflammation on the infant birth weight in a cohort of women without gestational diabetes.

\section{Methods}

We conducted this analysis within an ongoing prospective observational cohort study, in which women are recruited at the time of antepartum screening for gestational diabetes and followed into the postpartum period. The protocol for the ongoing study has been described in detail previously. ${ }^{11,12}$ In brief, following recruitment late in the second or early in the third trimester, all participants undergo a three-hour 100-g oral glucose tolerance test. Based on the results, the women are classified as having gestational diabetes (defined as two or more glucose values above the National Diabetes Data Group threshold), ${ }^{13}$ gestational impaired glucose tolerance (defined as only one value above the threshold) or normal glucose tolerance. ${ }^{11}$ Serum and plasma samples are obtained at the time of this test for assessment of biochemical factors. Obstetric outcomes are assessed at delivery. The protocol has been approved by the Mount Sinai Hospital Research Ethics Board, and all participants have provided written informed consent.

For the current analysis, we included participants who were identified as having either gestational impaired glucose tolerance or normal glucose tolerance (including those with an abnormal screening glucose challenge test). We excluded women with gestational diabetes because the glucose-lowering treatment (diet or insulin) that they would receive to reduce the risk of excessive fetal growth would obscure the associations between maternal factors and birth weight. The study population was further restricted to white, Asian and South Asian women because these are the groups for which Canadian-based ethnicityspecific centiles of birth weight have been established. ${ }^{14,15}$ Lastly, the analysis was restricted to women with term (37-41 weeks' gestation inclusive) singleton pregnancies, because both prematurity and twin pregnancy can affect infant growth.

\section{Biochemical analysis}

Fasting serum samples for biochemical analysis were obtained at the time of the oral glucose tolerance test. Fasting insulin levels were measured with the Roche Elecsys 1010 immunoassay analyzer and electrochemiluminescence immunoassay kit (Roche Diagnostics, Laval, Quebec).

Levels of total cholesterol, high-density lipoprotein (HDL) cholesterol and triglycerides were measured with the Roche Cobas 6000 c 501 analyzer (Roche Diagnostics, Laval). Levels of low-density lipoprotein (LDL) cholesterol were estimated with use of the Friedwald formula. Apolipoprotein B and A-I levels were measured with the BN ProSpec System (Siemens Healthcare Diagnostics, Mississauga, Ontario).

Total adiponectin levels were measured with an enzyme-linked immunosorbent assay (Millipore Corporation, Linco Research Inc., St. Charles, Missouri). High-sensitivity C-reactive protein levels were measured by means of endpoint nephelometry with use of the $\mathrm{BN}$ ProSpec Analyzer and $\mathrm{N}$ high-sensitivity Creactive protein reagent (Dade-Behring, Mississauga). Leptin levels were measured by means of enzyme-linked immunosorbent assay (model EZHL-80SK, Millipore Corporation, Linco Research Inc., St. Charles). The intraand intervariation coefficients for these assays have been reported previously. ${ }^{16}$

\section{Obstetric outcomes}

At delivery, data were collected on the length of gestation, the infant's sex, birth weight and Apgar scores, and the mode of delivery. A largefor-gestational-age infant was defined as one whose sex-specific birth weight for gestational age was above the 90th percentile of Canadian fetal growth curves for the relevant ethnic group (white, Asian or South Asian). ${ }^{14,15}$ Macrosomia was defined as a birth weight of $4000 \mathrm{~g}$ or higher.

\section{Statistical analysis}

We assessed maternal glycemia according to glucose tolerance status and area-under-the-glucosecurve on the oral glucose tolerance test. We tested continuous variables for normality of distribution, applying natural log-transformations of 
skewed variables where necessary. We stratified the study population into tertiles based on infant birth weight. We compared continuous variables using analysis of variance and categorical variables using the $\chi^{2}$ or Fisher exact test.

Multiple linear regression analysis of infant birth weight was performed, with adjustment for the following covariates: length of gestation, infant sex, maternal demographic factors (age, ethnicity, family history of diabetes), smoking status, anthropometric measures (body mass index [BMI] before pregnancy, and weight gain during pregnancy up to the time of the oral glucose tolerance test), glucose tolerance status, lipids (LDL, HDL and triglycerides), insulin, adipokines (adiponectin, leptin) and inflammatory proteins (C-reactive protein).

Logistic regression analysis of the dependent variable (large-for-gestational-age infant) was performed with use of the same covariates as in the multiple linear regression analysis, except for length of gestation and infant sex because both of these variables are considered when defining large-for-gestational-age infants.

Sensitivity analyses were performed in which the homeostasis model assessment of $\beta$-cell function and insulin resistance was used in place of fasting insulin, and the area-under-the glucose-curve was used in place of gestational impaired glucose tolerance. These sensitivity analyses were performed to assess robustness of the findings upon adjustment for $\beta$-cell function, insulin resistance and a continuous measure of glycemia.

All analyses were performed with the use of Statistical Analysis System 9.2 (SAS Institute, Cary, North Carolina).

\section{Results}

\section{Antepartum characteristics and obstetric outcomes}

Of the 472 women included in our study, 368 had normal glucose tolerance and 104 had gestational impaired glucose tolerance. Overall, the infants had a mean birth weight of $3481 \mathrm{~g}$ (standard deviation [SD] $493 \mathrm{~g}$ ), and 68 were large for gestational age at delivery.

Table 1 shows the antepartum characteristics and obstetric outcomes stratified by tertile of infant birth weight. The three groups differed in ethnicity, with the lowest tertile having the greatest proportion of Asian and South Asian women. As expected, BMI before pregnancy differed across the groups and was highest in the top tertile of birth weight. Consistent with this difference in BMI, the top tertile also exhibited higher levels of fasting glucose, insulin and triglycerides, and lower levels of adiponectin.

At delivery, the top tertile had the longest length of gestation and the largest proportion of cesarean deliveries. Although the proportion of infants with an Apgar score below 7 at one minute differed significantly between the three groups, the proportion with a score below 7 at five minutes did not. As expected, there were no instances of macrosomia or large-for-gestationalage infants in the lower two tertiles.

\section{Maternal factors associated with infant birth weight}

The unadjusted associations between maternal and fetal factors and birth weight are shown in Table 2. Length of gestation, male infant, BMI before pregnancy and weight gain during pregnancy up to the time of the oral glucose tolerance test were all associated with higher birth weight. South Asian ethnicity and adiponectin level were associated with lower birth weight. After adjustment for covariates, positive determinants of birth weight were length of gestation, male infant, BMI before pregnancy, weight gain during pregnancy up to the time of the oral glucose tolerance test and gestational impaired glucose tolerance. Leptin, adiponectin and C-reactive protein were each negatively associated with birth weight. This model explained $25.9 \%$ of the variance in birth weight.

On logistic regression analysis, the significant predictors of having a large-for-gestational-age infant, after adjustment for covariates, were Asian ethnicity (v. white) (OR 3.02, 95\% CI 1.25-7.27), BMI before pregnancy (OR 1.16, $95 \%$ CI $1.05-1.27$, per $1 \mathrm{~kg} / \mathrm{m}^{2}$ increase), weight gain during pregnancy up to the time of the oral glucose tolerance test (OR 1.12, 95\% CI 1.051.19 , per $1 \mathrm{~kg}$ increase) and leptin level (OR $0.50,95 \%$ CI $0.30-0.82$, per 1 SD change) (Figure 1, top panel). Because Asian women were found to have a greater risk of ethnicity-specific large-for-gestational-age infants compared with white women, we repeated the logistic regression analysis and included only the white women $(n=388)$. In this analysis, BMI before pregnancy (OR 1.13, 95\% CI 1.02-1.24, per $1 \mathrm{~kg} / \mathrm{m}^{2}$ increase), weight gain during pregnancy up to the time of the oral glucose tolerance test (OR $1.10,95 \%$ CI $1.03-1.18$, per $1 \mathrm{~kg}$ increase) and leptin level (OR 0.52, 95\% CI 0.29-0.91, per $1 \mathrm{SD}$ change) again emerged as significant predictors of large-for-gestational-age infants; being a former smoker (v. never smoking) also reached significance (OR 2.00, 95\% CI 1.02-3.93) (Figure 1 , bottom panel).

The sensitivity analyses identified the same independent predictors on multiple linear regres- 
Table 1: Characteristics and pregnancy outcomes of 472 women without gestational diabetes, by tertile of infant birth weight

\begin{tabular}{|c|c|c|c|c|}
\hline \multirow[b]{2}{*}{ Variable } & \multicolumn{3}{|c|}{ Tertile of infant birth weight; no. (\% of women)* } & \multirow[b]{2}{*}{$p$ valuet } \\
\hline & $\begin{array}{c}\text { Lowest tertile } \\
\text { [2020-3260 g] } \\
n=156\end{array}$ & $\begin{array}{c}\text { Middle tertile } \\
\text { [3260-3670 g] } \\
n=157\end{array}$ & $\begin{array}{c}\text { Highest tertile } \\
{[3670-5700 \mathrm{~g}]} \\
n=159\end{array}$ & \\
\hline \multicolumn{5}{|l|}{ At oral glucose tolerance test } \\
\hline Age, yr, mean (SD) & $33.6(4.0)$ & $34.5(4.3)$ & $33.6(4.0)$ & 0.08 \\
\hline No. of weeks of gestation, median (IQR) & $30(29-32)$ & $30(28-32)$ & $30(28-31)$ & 0.06 \\
\hline Ethnicity & & & & 0.009 \\
\hline White & $118(75.6)$ & $134(85.4)$ & $136(85.5)$ & \\
\hline Asian & $23(14.7)$ & $16(10.2)$ & $21(13.2)$ & \\
\hline South Asian & $15(9.6)$ & $7(4.5)$ & $2(1.3)$ & \\
\hline Family history of diabetes & $87(55.8)$ & $81(51.6)$ & $90(56.6)$ & 0.6 \\
\hline Parity & & & & 0.08 \\
\hline 0 & $94(60.3)$ & $78(49.7)$ & $79(49.7)$ & \\
\hline 1 & $52(33.3)$ & $61(38.9)$ & $56(35.2)$ & \\
\hline$>1$ & $10(6.4)$ & $18(11.5)$ & $24(15.1)$ & \\
\hline Previous gestational diabetes & $5 \quad(3.2)$ & $8(5.1)$ & $11(6.9)$ & 0.3 \\
\hline Smoking status & & & & 0.9 \\
\hline Never smoked & $103(66.0)$ & $109(69.4)$ & $105(66.0)$ & \\
\hline Former smoker & $49(31.4)$ & $44(28.0)$ & $51(32.1)$ & \\
\hline Current smoker & 4 (2.6) & $4(2.6)$ & $3(1.9)$ & \\
\hline BMI before pregnancy, $\mathrm{kg} / \mathrm{m}^{2}$, median (IQR) & $22.6(20.7-25.4)$ & $22.6(20.8-25.8)$ & $23.6(22.3-27.4)$ & $<0.001$ \\
\hline $\begin{array}{l}\text { Weight gain during pregnancy up to the oral } \\
\text { glucose tolerance test, } \mathrm{kg} \text {, mean (SD) }\end{array}$ & $10.8(5.4)$ & $11.6(4.9)$ & $11.6(5.7)$ & 0.3 \\
\hline Weight gain per week of gestation, kg/wk, mean (SD) & $0.36(0.18)$ & $0.38(0.16)$ & $0.39(0.18)$ & 0.3 \\
\hline Fasting glucose, mmol/L, mean (SD) & $4.4(0.4)$ & $4.4(0.4)$ & $4.6(0.5)$ & 0.001 \\
\hline Area-under-glucose-curve, mean (SD) & $21.1(2.6)$ & $21.4(3.0)$ & $21.5(2.8)$ & 0.5 \\
\hline Glucose tolerance in pregnancy & & & & 0.08 \\
\hline Normal & $131(84.0)$ & $117(74.5)$ & $120(75.5)$ & \\
\hline Impaired & $25(16.0)$ & $40(25.5)$ & $39(24.5)$ & \\
\hline Fasting insulin, pmol/L, median (IQR) & $57(39-79)$ & $53(36-71)$ & $65(42-94)$ & $<0.001$ \\
\hline Total cholesterol, mmol/L, mean (SD) & $6.48(1.25)$ & $6.55(1.23)$ & $6.39(1.15)$ & 0.5 \\
\hline LDL cholesterol, mmol/L, mean (SD) & $3.72(1.17)$ & $3.72(1.12)$ & $3.6(1.04)$ & 0.5 \\
\hline HDL cholesterol, mmol/L, mean (SD) & $1.73(0.36)$ & $1.72(0.37)$ & $1.66(0.34)$ & 0.2 \\
\hline Triglycerides, mmol/L, mean (SD) & $2.25(0.72)$ & $2.46(0.75)$ & $2.49(0.66)$ & 0.006 \\
\hline Apolipoprotein B, g/L, mean (SD) & $1.28(0.31)$ & $1.30(0.30)$ & $1.29(0.28)$ & 0.7 \\
\hline Apolipoprotein A-I, mmol/L, mean (SD) & $2.10(0.28)$ & $2.10(0.32)$ & $2.07(0.26)$ & 0.5 \\
\hline C-reactive protein, mg/L, median (IQR) & $4.5(2.5-7.6)$ & $4.1(2.0-7.5)$ & $3.8(2.3-7.1)$ & 0.7 \\
\hline Adiponectin, $\mu \mathrm{g} / \mathrm{mL}$, median (IQR) & $8.2(6.3-10.0)$ & $8.6(6.7-10.4)$ & $7.5 \quad(6.0-9.2)$ & 0.02 \\
\hline Leptin, ng/mL, median (IQR) & $34.5(25.3-46.4)$ & $31.7(19.8-45.8)$ & $33.4(23.1-46.2)$ & 0.7 \\
\hline \multicolumn{5}{|l|}{ At delivery } \\
\hline Length of gestation, wk, mean (SD) & $38.6(1.1)$ & $39.2(1.0)$ & $39.6(1.1)$ & $<0.001$ \\
\hline Infant sex, male/female, \% & $40 / 60$ & $50 / 50$ & $52 / 48$ & 0.07 \\
\hline Infant with Apgar score $<7$ at $1 \mathrm{~min}$ & $12(7.8)$ & $2(1.3)$ & $10(6.3)$ & 0.02 \\
\hline Infant with Apgar score $<7$ at $5 \mathrm{~min}$ & 0 & 0 & $2(1.3)$ & 0.1 \\
\hline Cesarean delivery & $49(31.4)$ & $49(31.2)$ & $75(47.2)$ & 0.003 \\
\hline Infant with macrosomia & 0 & 0 & $61(38.4)$ & $<0.001$ \\
\hline Large-for-gestational-age infant & 0 & 0 & $68(42.8)$ & $<0.001$ \\
\hline
\end{tabular}


sion and logistic regression as those in Table 2 and Figure 1 (top panel), respectively.

\section{Interpretation}

Our study yielded three key findings. First, the strongest determinant of infant birth weight and of having a large-for-gestational-age infant was maternal adiposity, with BMI before pregnancy and weight gain during pregnancy emerging as the strongest predictors for both outcomes in the fully adjusted analyses. This finding is consistent with those of previous studies, which suggests the central role of maternal weight as a determinant of birth weight. ${ }^{17-19}$ Although the underlying mechanisms remain unclear, shared genetic factors may be relevant to both maternal adiposity and fetal growth.

Obesity is associated with pathologic sequelae, including chronic inflammation (increased Creactive protein level) and adipocyte dysregulation (increased leptin and low adiponectin levels). ${ }^{20} \mathrm{In}$ this context, the second key observation of our study pertains to the relative contributions of these obesity-related circulating factors. Specifically, we found leptin to be a significant negative predictor of both birth weight and large-for-gestational-age infant after adjustment for covariates, including BMI before pregnancy and weight gain during pregnancy. An inverse relation between leptin level and infant birth weight has been observed in some, though not all, previous studies. ${ }^{21-24}$ Of note, Verhaeghe and colleagues found that, compared with glucose, insulin, adiponectin and tumour necrosis factor- $\alpha$, leptin was the mediator that was most strongly (and negatively) associated with birth weight. ${ }^{23}$ Placental dysfunction has been suggested as a unifying mechanism underlying both fetal growth restriction and elevated maternal leptin levels (through increased placental production of leptin), although this model remains speculative. ${ }^{24}$

Like leptin, C-reactive protein also emerged as a negative determinant of infant birth weight in our multiple linear regression analysis. In the Hyperglycemia and Adverse Pregnancy Outcome Study, an inverse relation between C-reactive pro-

Table 2: Change in infant birth weight in relation to maternal and fetal factors*

\begin{tabular}{|c|c|c|c|}
\hline \multirow{3}{*}{$\begin{array}{l}\text { Variable } \\
\text { Length of gestation, wk }\end{array}$} & \multicolumn{3}{|c|}{ Change in infant birth weight, g $(95 \% \mathrm{Cl})$} \\
\hline & \multicolumn{2}{|r|}{ Crude } & Adjusted $\dagger$ \\
\hline & 166.65 & (129.99 to 203.32$)$ & 164.85 (127.50 to 202.22$)$ \\
\hline Male infant & 115.76 & (26.97 to 204.54$)$ & (55.55 to 223.11$)$ \\
\hline Age, yr & 2.24 & $(-8.60$ to 13.08$)$ & $-0.26 \quad(-10.49$ to 9.97$)$ \\
\hline \multicolumn{4}{|l|}{ Ethnicity (v. white) } \\
\hline Asian & \multicolumn{2}{|c|}{$-102.78(-236.45$ to 30.89$)$} & $-28.48(-169.09$ to 112.13$)$ \\
\hline South Asian & \multicolumn{2}{|c|}{$-322.67(-523.73$ to -121.62$)$} & $-165.12(-375.24$ to 44.99$)$ \\
\hline Family history of diabetes & \multicolumn{2}{|c|}{$27.66 \quad(-61.96$ to 117.28$)$} & $15.31 \quad(-69.47$ to 100.09$)$ \\
\hline \multicolumn{4}{|l|}{ Smoking status (v. never smoked) } \\
\hline Former & \multicolumn{2}{|c|}{$52.64 \quad(-44.17$ to 149.46$)$} & $45.84 \quad(-44.90$ to 136.58$)$ \\
\hline Current & \multicolumn{2}{|c|}{$-79.35(-375.10$ to 216.40$)$} & $-187.36(-464.34$ to 89.62$)$ \\
\hline BMI before pregnancy, $\mathrm{kg} / \mathrm{m}^{2}$ & 15.22 & (5.66 to 24.78$)$ & $22.45 \quad$ (9.66 to 35.25$)$ \\
\hline $\begin{array}{l}\text { Weight gain during pregnancy up to } \\
\text { oral glucose tolerance test, } \mathrm{kg}\end{array}$ & 9.49 & (1.11 to 17.87$)$ & $19.15 \quad$ (9.93 to 28.37$)$ \\
\hline $\begin{array}{l}\text { Impaired glucose tolerance in } \\
\text { pregnancy (v. normal glucose tolerance) }\end{array}$ & 100.29 & (-7.01 to 207.59$)$ & 120.37 (19.90 to 220.84$)$ \\
\hline Fasting insulin, $\mathrm{pmol} / \mathrm{L}$ & 0.63 & $(-0.06$ to 1.32$)$ & $0.16 \quad(-0.53$ to 0.85$)$ \\
\hline LDL cholesterol, mmol/L & \multicolumn{2}{|c|}{-15.22 ( -55.49 to 25.05$)$} & $-6.79 \quad(-46.98$ to 33.39$)$ \\
\hline HDL cholesterol, mmol/L & \multicolumn{2}{|c|}{$-120.54(-244.42$ to 3.35$)$} & $-57.16(-189.42$ to 75.09$)$ \\
\hline Triglycerides, mmol/L & 61.11 & $(-1.18$ to 123.40$)$ & $-1.59 \quad(-70.67$ to 67.49$)$ \\
\hline C-reactive protein, $\mathrm{mg} / \mathrm{L}$ & -3.68 & $(-10.98$ to 3.63$)$ & $(-14.88$ to -0.47$)$ \\
\hline Adiponectin, $\mu \mathrm{g} / \mathrm{mL}$ & -21.53 & $(-37.67$ to -5.38$)$ & $(-36.62$ to -2.07$)$ \\
\hline Leptin, per ng/mL & -0.81 & $(-2.79$ to 1.16$)$ & $(-6.23$ to -1.60$)$ \\
\hline \multicolumn{4}{|c|}{$\begin{array}{l}\text { Note: } \mathrm{BMI}=\text { body mass index, } \mathrm{Cl}=\text { confidence interval, } \mathrm{HDL}=\text { high-density lipoprotein, } \mathrm{LDL}=\text { low-density lipoprotein. } \\
{ }^{*} \text { Change in infant birth weight per unit change in indicated variable. For example, infant birth weight increased by } 164.85 \mathrm{~g} \\
\text { per additional week of gestation after adjustment for the other variables. } \\
\text { tAdjusted for all other variables listed. }\end{array}$} \\
\hline
\end{tabular}


tein level and birth weight was seen after adjustment for covariates, including maternal BMI. ${ }^{25}$ The negative associations of leptin and C-reactive protein with birth weight became apparent in our study only after adjustment for covariates, including BMI before pregnancy. Thus, despite the elevated circulating levels of leptin and C-reactive protein in the setting of obesity (which itself is the primary determinant of increased birth weight), both of these proteins were found to be independently associated with decreased birth weight. Although the mechanisms are unclear, our data suggest that these factors may play a role in attenuating the pro-macrosomia effects of maternal

\begin{tabular}{l} 
Variable* \\
\hline All women \\
Age, yr \\
Asian ethnicity (v. white) \\
South Asian ethnicity (v. white) \\
Family history of diabetes mellitus \\
Former smoker ( $\mathrm{v}$. never smoked) \\
Current smoker ( $\mathrm{v}$. never smoked) \\
BMI before pregnancy, kg/m² \\
Weight gain during pregnancy \\
up to oral glucose tolerance test, $\mathrm{kg}$ \\
Impaired glucose tolerance \\
in pregnancy \\
Fasting insulin, pmol/L \\
LDL cholesterol, mmol/L \\
HDL cholesterol, mmol/L \\
Triglycerides, mmol/L \\
C-reactive protein, mg/L \\
Adiponectin, $\mu \mathrm{g} / \mathrm{mL}$ \\
Leptin, ng/mL
\end{tabular}

White women

Age, yr

Family history of diabetes mellitus

Former smoker (v. never smoked)

Current smoker (v. never smoked)

BMI before pregnancy, $\mathrm{kg} / \mathrm{m}^{2}$

Weight gain during pregnancy up to oral glucose tolerance test, $\mathrm{kg}$ Impaired glucose tolerance in pregnancy

Fasting insulin, pmol/L LDL cholesterol, $\mathrm{mmol} / \mathrm{L}$ HDL cholesterol, $\mathrm{mmol} / \mathrm{L}$ Triglycerides, $\mathrm{mmol} / \mathrm{L}$ C-reactive protein, $\mathrm{mg} / \mathrm{L}$ Adiponectin, $\mu \mathrm{g} / \mathrm{mL}$ Leptin, $\mathrm{ng} / \mathrm{mL}$
Unadjusted* odds Adjusted* odds ratio $(95 \% \mathrm{Cl}) \quad$ ratio $(95 \% \mathrm{Cl})$

$1.02(0.96-1.08)$

$1.97(1.01-3.83)$

$0.59(0.13-2.57)$

$1.41(0.83-2.38)$

$1.53(0.89-2.62)$

$1.54(0.32-7.38)$

$1.04(0.99-1.10)$

$1.05(1.00-1.10)$

$1.59(0.89-2.82)$

$1.09(0.89-1.33)$

$0.80(0.61-1.05)$

$0.89(0.69-1.15)$

$1.26(0.98-1.62)$

$0.79(0.57-1.10)$

$0.68(0.50-0.92)$

0.85 (0.63-1.13)

$1.00(0.93-1.07)$
$3.02(1.25-7.27)$
$1.45(0.28-7.44)$
$1.62(0.88-2.98)$
$1.82(0.99-3.37)$
$1.75(0.29-10.69)$
$1.16(1.05-1.27)$
$1.12(1.05-1.19)$
$1.36(0.71-2.61)$
$0.97(0.70-1.34)$
$0.98(0.72-1.34)$
$0.99(0.70-1.39)$
$0.98(0.70-1.38)$
$0.75(0.50-1.12)$
$0.79(0.55-1.13)$
$0.50(0.30-0.82)$

Decreased I Increased $\longleftarrow$ risk risk $\longrightarrow$

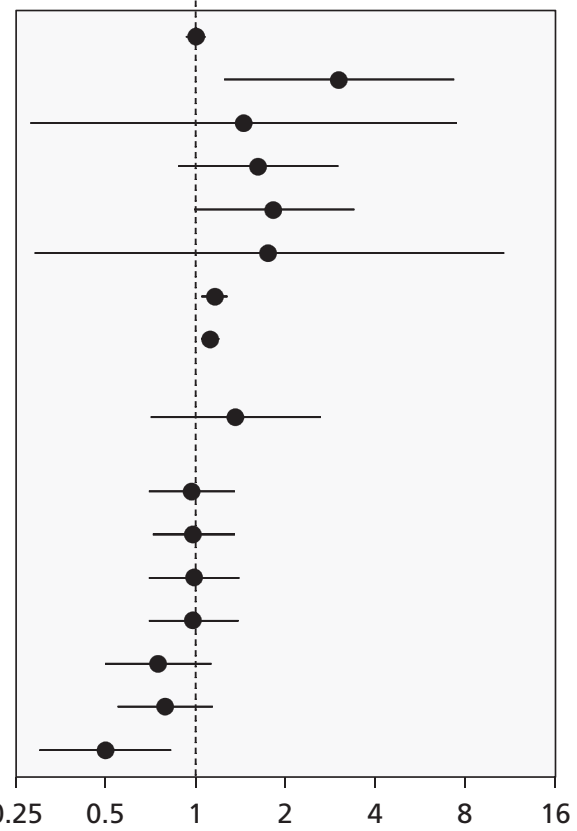

1.02 (0.95-1.09)

$1.56(0.85-2.85)$

$1.75(0.96-3.20)$

$0.81(0.37-8.82)$

$1.04(0.99-1.10)$

$1.06(1.00-1.11)$

$1.34(0.68-2.65)$

$1.06(0.85-1.32)$

$0.85(0.62-1.16)$

$0.82(0.60-1.10)$

$1.33(1.00-1.77)$

$0.80(0.55-1.16)$

$0.64(0.45-0.92)$

$0.88(0.63-1.23)$
$1.01(0.93-1.10)$

$1.65(0.83-3.28)$

$2.00(1.02-3.93)$

$1.79(0.29-10.83)$

$1.13(1.02-1.24)$

1.10 (1.03-1.18)

$1.34(0.62-2.90)$

$0.90(0.54-1.48)$

$0.98(0.69-1.38)$

$1.03(0.69-1.52)$

$1.07(0.73-1.58)$

$0.69(0.43-1.12)$

$0.70(0.46-1.08)$

$0.52(0.29-0.91)$

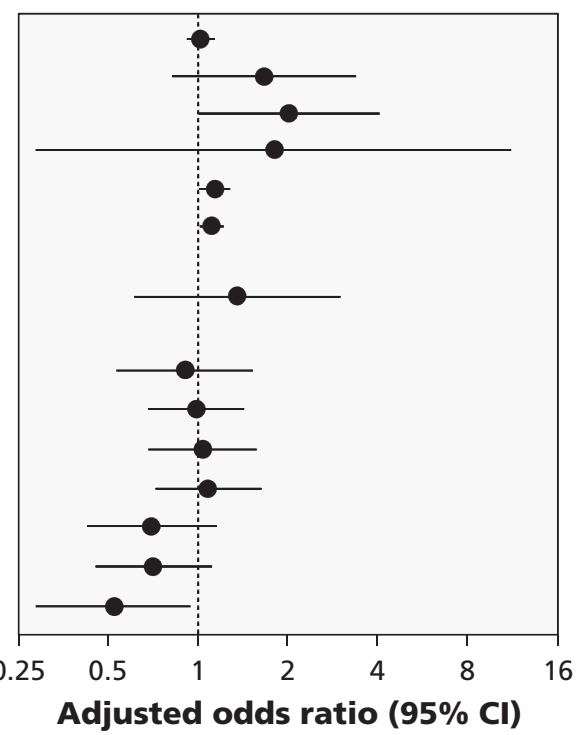

Figure 1: Independent predictors of having a large-for-gestational-age infant among women without gestational diabetes, in the entire study population (top panel) and among white women only (bottom panel). An odds ratio greater than 1.00 is associated with an increased risk of having a large-for-gestational-age infant. Odds ratios for continuous variables are expressed per 1 standard deviation change in the indicated variable, except for age (expressed per year increase), body mass index (BMI) before pregnancy (expressed per $1 \mathrm{~kg} / \mathrm{m}^{2}$ increase) and weight gain during pregnancy (expressed per $1 \mathrm{~kg}$ increase). For example, the adjusted odds ratio for having a large-for-gestational-age infant increased by 1.16 per $1 \mathrm{~kg} / \mathrm{m}^{2}$ increase in BMI before pregnancy. *Each variable was adjusted for all others in the list. $\mathrm{Cl}$ = confidence interval, $\mathrm{HDL}=$ high-density lipoprotein, $\mathrm{LDL}=$ low-density lipoprotein. 
adiposity. Overall, coupled with the inverse relation between total adiponectin level and birth weight, these data suggest a model in which fetal growth is influenced both positively and negatively by complex interplay between maternal adiposity and its associated circulating factors.

The third key finding from our study is that, although gestational impaired glucose tolerance was an independent predictor of birth weight in the multiple linear regression analysis, its impact was relatively modest compared with that of BMI before pregnancy, weight gain during pregnancy up to the time the oral glucose tolerance test and the leptin level. Moreover, unlike the latter three factors, gestational impaired glucose tolerance was not a significant independent predictor of having a large-for-gestational-age infant. Similarly, none of the lipid measures was independently associated with birth weight or large-forgestational-age infant. These data suggest that maternal weight and its associated circulating factors have a greater impact on infant birth weight than do mild glucose intolerance and lipid levels in women without gestational diabetes.

This concept is consistent with findings from two recent studies addressing the relative effects of maternal obesity and glycemia on fetal growth. In a re-analysis of data from the Hyperglycemia and Adverse Pregnancy Outcome Study, Ryan recently showed that maternal BMI had a greater impact on the odds of having a large-for-gestational-age infant than did maternal glucose in all but the highest category of glycemia. ${ }^{26}$ In addition, in a study involving Spanish women, Ricart and colleagues found that BMI before pregnancy commanded a much higher population-attributable risk for macrosomia than did gestational hyperglycemia. ${ }^{17}$ Our study further extends this discussion by showing that both BMI before pregnancy and weight gain during pregnancy had a greater independent impact on the risk of excessive fetal growth than did glycemia in women without gestational diabetes.

This emerging concept regarding the relative importance of maternal obesity versus glycemia may hold implications for the current debate regarding the lowering of diagnostic thresholds for gestational diabetes on antepartum glucose tolerance testing. ${ }^{26,27}$ Specifically, among the additional women who will be identified as having gestational diabetes owing to this change, their mild glucose intolerance may play a comparatively modest role in determining risk of macrosomia. In this context, it may instead be more prudent to target maternal obesity, the prevalence of which has risen dramatically in recent years. ${ }^{10}$

\section{Strengths and limitations}

A major strength of our analysis is that it evaluated simultaneously the effect of several maternal metabolic factors, including glucose tolerance, adiposity, and fasting levels of insulin, lipids, adipokines and inflammatory proteins. This approach enabled the assessment of the factors' independent associations with infant birth weight after adjustment for one another.

One limitation of our study is that we did not evaluate the high-molecular-weight form of adiponectin. Although total and high-molecularweight adiponectin are generally highly correlated, the high-molecular-weight form may be the specific maternal mediator affecting fetal growth. ${ }^{28}$ Another limitation is that weight before pregnancy was determined by patient recall and hence may have been subject to bias. In addition, weight gain during pregnancy was determined only up to the time of the oral glucose tolerance test and not across the total length of gestation. However, the impact of this limitation should be modest, because changes in maternal weight in the first and second trimesters have been shown to have a much greater influence on birth weight than changes in the third trimester. ${ }^{29}$

\section{Conclusion}

Among women without gestational diabetes, the strongest metabolic determinant of macrosomia was maternal adiposity, as reflected by BMI before pregnancy and weight gain during pregnancy up to the time of the oral glucose tolerance test. Furthermore, after adjustment for these attributes, weight-related circulating factors (particularly leptin) emerged as negative independent predictors of having a large-for-gestational-age infant. In contrast, glucose intolerance and lipid levels had a modest effect in this cohort.

In the context of the current obesity epidemic, these data support the importance of targeting healthy body weight in young women as a strategy for reducing the risk of excessive fetal growth and infant macrosomia. Furthermore, these findings suggest that, in the care of overweight or obese women in pregnancy, closer monitoring of weight gain during pregnancy may be warranted.

\section{References}

1. Pedersen J. Diabetes and pregnancy: blood sugar of newborn infants [dissertation]. Copenhagen (Denmark): Danish Science Press; 1952. p.230.

2. Freinkel N. Banting lecture 1980. Of pregnancy and progeny. Diabetes 1980;29:1023-35.

3. Lindsay RS. Many HAPO returns: maternal glycemia and neonatal adiposity: new insights from the Hyperglycemia and Adverse Pregnancy Outcomes (HAPO) study. Diabetes 2009;58: 302-3.

4. Sermer M, Naylor CD, Gare DJ, et al. Impact of increasing carbohydrate intolerance on maternal-fetal outcomes in 3637 wo- 
men without gestational diabetes. The Toronto Tri-Hospital Gestational Diabetes Project. Am J Obstet Gynecol 1995; 173:146-56.

5. Metzger BE, Lowe LP, Dyer AR, et al.; HAPO Study Cooperative Research Group. Hyperglycemia and adverse pregnancy outcomes. N Engl J Med 2008;358:1991-2002.

6. Knopp RH, Magee MS, Walden CE, et al. Prediction of infant birth weight by GDM screening tests. Importance of plasma triglyceride. Diabetes Care 1992;15:1605-13.

7. Di Cianni G, Miccoli R, Volpe L, et al. Maternal triglyceride levels and newborn weight in pregnant women with normal glucose tolerance. Diabet Med 2005;22:21-5.

8. Metzger BE, Gabbe SG, Persson B, et al. International Association of Diabetes and Pregnancy Study Groups recommendations on the diagnosis and classification of hyperglycemia in pregnancy. Diabetes Care 2010;33:676-82.

9. Catalano PM, Hauguel-De Mouzon S. Is it time to revisit the Pedersen hypothesis in the face of the obesity epidemic? Am J Obstet Gynecol 2011;204:479-87.

10. Kanagalingam MG, Forouhi NG, Greer IA, et al. Changes in booking body mass index over a decade: retrospective analysis from a Glasgow Maternity Hospital. BJOG 2005;112:1431-3.

11. Retnakaran R, Qi Y, Sermer M, et al. Glucose intolerance in pregnancy and future risk of pre-diabetes or diabetes. Diabetes Care 2008;31:2026-31.

12. Retnakaran R, Qi Y, Connelly PW, et al. Low adiponectin concentration during pregnancy predicts postpartum insulin resistance, beta-cell dysfunction and fasting glycaemia. Diabetologia 2010;53:268-76.

13. National Diabetes Data Group. Classification and diagnosis of diabetes mellitus and other categories of glucose intolerance. Diabetes 1979;28:1039-57.

14. Kramer MS, Platt RW, Wen SW, et al.; Fetal/Infant Health Study Group of the Canadian Perinatal Surveillance System. A new and improved population-based Canadian reference for birthweight for gestational age. Pediatrics 2001;108:E35-41.

15. Kierans WJ, Kramer MS. Wilkins R, et al. Charting birth out come in British Columbia: determinants of optimal health and ultimate risk - an expansion and update. Victoria (BC): British Columbia Vital Statistics Agency; 2003. Available: www.vs.gov .bc.ca/stats/features/index.html (accessed 2011 Mar. 17)

16. Retnakaran R, Qi Y, Sermer M, et al. The postpartum cardiovascular risk factor profile of women with isolated hyperglycemia at 1-hour on the oral glucose tolerance test in pregnancy. Nutr Metab Cardiovasc Dis 2011;21:706-12.

17. Ricart W, López J, Mozas J, et al.; Spanish Group for the Study of the Impact of Carpenter and Coustan GDM Thresholds. Body mass index has a greater impact on pregnancy outcomes than gestational hyperglycemia. Diabetologia 2005;48:1736-42.

18. HAPO Study Cooperative Research Group. Hyperglycaemia and Adverse Pregnancy Outcome (HAPO) Study: associations with maternal body mass index. BJOG 2010;117:575-84

19. Yu CK, Teoh T, Robinson S. Obesity in pregnancy. BJOG 2006; 113:1117-25.

20. Rasouli N, Kern PA. Adipocytokines and the metabolic complications of obesity. J Clin Endocrinol Metab 2008;93:S64-73.

21. Papastefanou I, Samolis S, Panagopoulos P, et al. Correlation between maternal first trimester plasma leptin levels and birth weight among normotensive and preeclamptic women. J Matern Fetal Neonatal Med 2010;23:1435-43.

22. Mise H, Yura S, Itoh $\mathrm{H}$, et al. The relationship between maternal plasma leptin levels and fetal growth restriction. Endocr J 2007; 54:945-51.

23. Verhaeghe J, van Bree R, Van Herck E. Maternal body size and birthweight: Can insulin or adipokines do better? Metabolism 2006;55:339-44.

24. Pighetti M, Tommaselli GA, D'Elia A, et al. Maternal serum and umbilical cord blood leptin concentrations with fetal growth restriction. Obstet Gynecol 2003;102:535-43.

25. Lowe LP, Metzger BE, Lowe WL Jr, et al.; HAPO Study Cooperative Research Group. Inflammatory mediators and glucose in pregnancy: results from a subset of the Hyperglycemia and Adverse Pregnancy Outcome (HAPO) Study. J Clin Endocrinol Metab 2010;95:5427-34

26. Ryan EA. Diagnosing gestational diabetes. Diabetologia 2011; 54:480-6.

27. Long H. Diagnosing gestational diabetes: Can expert opinions replace scientific evidence? Diabetologia 2011;54:2211-3.

28. Ong GK, Hamilton JK, Sermer M, et al. Maternal serum adiponectin and infant birthweight: the role of adiponectin isoform distribution. Clin Endocrinol (Oxf) 2007;67:108-14.

29. Brown JE, Murtaugh MA, Jacobs DR, et al. Variation in newborn size according to pregnancy weight change by trimester. Am J Clin Nutr 2002;76:205-9.

Affiliations: From the Leadership Sinai Centre for Diabetes (Retnakaran, Ye, Hanley, Zinman), the Division of Obstetrics and Gynecology (Sermer) and the Samuel Lunenfeld Research Institute (Zinman), Mount Sinai Hospital; the Division of Endocrinology (Retnakaran, Hanley, Connelly, Zinman, Hamilton) and the Department of Nutritional Sciences (Hanley), University of Toronto; the Keenan Research Centre (Connelly), Li Ka Shing Knowledge Institute, St. Michael's Hospital; and the Division of Endocrinology (Hamilton), Hospital for Sick Children, Toronto, Ont.

Contributors: Ravi Retnakaran designed the analysis plan, researched the data and wrote the manuscript. Chang Ye performed the statistical analyses. Ravi Retnakaran, Anthony Hanley, Philip Connelly, Mathew Sermer, Bernard Zinman and Jill Hamilton were involved in the design and implementation of the overall study. All of the authors contributed to critical revision of the manuscript and approved the final version submitted for publication.

Funding: This study was supported by operating grants from the Canadian Institutes of Health Research (CIHR) (grant nos. MOP-89831 and MOP-84206). The study sponsor had no role in the design of the study, the collection, analysis or interpretation of data, the writing of the report or the decision to submit the article for publication.

Acknowledgements: The authors thank Mount Sinai Hospital's Department of Pathology and Laboratory Medicine and Patient Care Services.

Ravi Retnakaran is supported by a CIHR Clinical Research Initiative New Investigator Award, Canadian Diabetes Association Clinician Scientist incentive funding and an Ontario Ministry of Research and Innovation Early Researcher Award. Anthony Hanley holds a Tier II Canada Research Chair in Diabetes Epidemiology. Bernard Zinman holds the Sam and Judy Pencer Family Chair in Diabetes Research at Mount Sinai Hospital and University of Toronto. 